\title{
EFFICIENT ROUTEING IN POISSON SMALL-WORLD NETWORKS
}

\author{
M. DRAIEF, ${ }^{*}$ University of Cambridge \\ A. GANESH, ${ }^{* *}$ Microsoft Research
}

\begin{abstract}
In a recent paper, Kleinberg (2000) considered a small-world network model consisting of a $d$-dimensional lattice augmented with shortcuts. The probability of a shortcut being present between two points decays as a power, $r^{-\alpha}$, of the distance, $r$, between them. Kleinberg showed that greedy routeing is efficient if $\alpha=d$ and that there is no efficient decentralised routeing algorithm if $\alpha \neq d$. The results were extended to a continuum model by Franceschetti and Meester (2003). In our work, we extend the result to more realistic models constructed from a Poisson point process wherein each point is connected to all its neighbours within some fixed radius, and possesses random shortcuts to more distant nodes as described above.
\end{abstract}

Keywords: Small world; routeing

2000 Mathematics Subject Classification: Primary 60D05; 68W15

Secondary 05C80

\section{Introduction}

A classical random graph model introduced by Erdős and Rényi (1959) consists of $n$ nodes, with the edge between any pair of vertices being present with probability $p(n)$, independent of other pairs. Recently, there has been considerable interest in alternative models where the nodes are given coordinates in a Euclidean space, and the probability of an edge between a pair of nodes $u$ and $v$ is given by a function $g(\cdot)$ of the distance, $r(u, v)$, between the nodes; edges between different node pairs are independent. Such 'random connection' or 'spatial random graph' models and variants thereof arise, for instance, in the study of wireless communications networks.

The 'small-world phenomenon' (the principle that all people are linked by short chains of acquaintances), which has long been a matter of folklore, was inaugurated as an area of experimental study in the social sciences through the pioneering work of Milgram (1967). Recent works have suggested that the phenomenon is pervasive in networks arising in nature and technology, and motivated interest in mathematical models of such networks. While ErdôsRényi random graphs possess the property of having a small diameter (smaller than logarithmic in the number of nodes, above the connectivity threshold for $p(n)$ ), they are not good models for social networks because of the independence assumption. On the other hand, spatial random graphs are better at capturing clustering because of the implicit dependence between edges induced by the connection function $g(\cdot)$.

Received 26 July 2005; revision received 27 March 2006.

* Postal address: Statistical Laboratory, Centre for Mathematical Sciences, University of Cambridge, Wilberforce Road, Cambridge CB3 0WB, UK. Email address: m.draief@statslab.cam.ac.uk

** Postal address: Microsoft Research, 7 J J Thomson Avenue, Cambridge CB3 0FB, UK.

Email address: ajg@microsoft.com 
Watts and Strogatz (1967) conducted a set of re-wiring experiments on graphs, and observed that by re-wiring a few random links in finite lattices, the average path length was reduced drastically (approaching that of random graphs). This led them to propose a model of 'smallworld graphs' which essentially consisted of a lattice augmented with random links acting as shortcuts, which played an important role in shrinking the average path link. By the length of a path we mean the number of edges in it, and distance refers to graph distance (length of shortest path) unless otherwise specified.

The diameter of the Watts-Strogatz model in the one-dimensional case was obtained by Barbour and Reinert (2001). Benjamini and Berger (2001) considered a variant of this onedimensional model wherein the shortcut between any pair of nodes, instead of being present with constant probability, is present with probability given by a connection function $g(\cdot)$; they specifically considered connection functions of the form $g(r) \sim \beta r^{-\alpha}$, where $\beta$ and $\alpha$ are given constants and $r(u, v)$ is the graph distance between $u$ and $v$ in the underlying lattice (i.e. the $L_{1}$ distance).

The general $d$-dimensional version of this model, on the finite lattice with $n^{d}$ points, was studied by Coppersmith et al. (2002). They showed that the diameter of the graph is

$$
\begin{array}{ll}
\Theta\left(\frac{\log n}{\log \log n}\right) & \text { if } \alpha=d, \\
\text { at most polylogarithmic in } n & \text { if } d<\alpha<2 d, \\
\text { at least polynomial in } n & \text { if } \alpha>2 d,
\end{array}
$$

where $X_{n}=\Theta\left(a_{n}\right)$ as $n \rightarrow \infty$ if for all $\delta>0$ there exist $c_{\delta}>0$ and $C_{\delta}>0$ such that, for all $n \geq n_{0}\left(\right.$ and some $\left.n_{0}\right)$,

$$
\mathrm{P}\left(c_{\delta} \leq \frac{X_{n}}{a_{n}} \leq C_{\delta}\right)>1-\delta .
$$

Finally, it was shown by Benjamini et al. (2004) that the diameter is a constant if $\alpha<d$.

The sociological experiments of Milgram demonstrated not only that there is a short chain of acquaintances between strangers but also that they are able to find such chains. What sort of graph models have this property? Specifically, when can decentralised routeing algorithms (which we define later) find a short path between arbitrary source and destination nodes?

This question was addressed by Kleinberg (2000) for the class of finite $d$-dimensional lattices augmented with shortcuts where the probability of a shortcut being present between two nodes decays as a power, $r^{-\alpha}$, of the distance, $r$, between them. Kleinberg showed that greedy routeing is efficient if $\alpha=d$ and that there is no efficient decentralised routeing algorithm if $\alpha \neq d$. The results were extended to a continuum model by Franceschetti and Meester (2003). Note that these results show that decentralised algorithms cannot find short routes when $\alpha \neq d$, even though such routes are present for $\alpha<2 d$, by the results of Benjamini et al. (2004) and Coppersmith et al. (2002); when $\alpha>2 d$, no short routes are present.

\section{Our model}

In this work, we consider a model constructed from a Poisson point process on a finite square, wherein each point is connected to all its neighbours within some fixed radius, and possesses random shortcuts to more distant nodes. We can express this more precisely as follows.

- We consider a sequence of graphs indexed by $n \in \mathbb{N}$.

- Nodes form a Poisson process of rate 1 on the square $[0, \sqrt{n}]^{2}$. 
- Each node $x$ is linked to all nodes that are a distance less than $r_{n}=\sqrt{c \log n}$ away, for a sufficiently large constant $c$. In particular, if $c>1 / \pi$ then this graph is connected with high probability (w.h.p.), meaning with probability going to 1 as $n$ tends to infinity; see Penrose (2003). These links are referred to as local edges and the corresponding nodes as the local contacts of $x$.

- For two nodes $u$ and $v$ such that $r(u, v)>\sqrt{c \log n}$, the edge $(u, v)$ is present with probability $a_{n} r(u, v)^{-\alpha} \wedge 1$. Such edges are referred to as shortcuts. The parameter $a_{n}$ is chosen so that the expected number of shortcuts per node is equal to some specified constant, $\bar{d}$.

The objective is to route a message from an arbitrary source node $s$ to an arbitrary destination $t$ using a small number of hops. We are interested in decentralised routeing algorithms, which do not require global knowledge of the graph topology. It is assumed throughout that each node knows its location (coordinates) on the plane, the location of all its neighbours, both local and shortcut, and the location of the destination $t$. We show that efficient decentralised routeing is possible only if $\alpha=2$. More precisely, we show the following.

- For $\alpha=2$, there is a greedy decentralised algorithm to route a message from source to destination in $O\left(\log ^{2} n\right)$ hops.

- For $\alpha<2$, any decentralised routeing needs more than $n^{\gamma}$ hops on average, for any $\gamma$ such that $\gamma<(2-\alpha) / 6$.

- For $\alpha>2$, any decentralised routeing needs more than $n^{\gamma}$ hops on average, for any $\gamma<(\alpha-2) / 2(\alpha-1)$.

As noted by Kleinberg (2000) for the lattice model, the case $\alpha=2$ corresponds to a 'scale-free' network, i.e. the expected number of shortcuts from a node $x$ to nodes which lie between a distance $r$ and a distance $2 r$ from it is the same for any $r$. In both Kleinberg's model and our model, the scale invariance is cut off at very small and very large distances because the graph is on a finite number of nodes. Full scale invariance can be observed in the continuum model of Franceschetti and Meester (2003) which is defined on the infinite plane (in two dimensions). They showed that scale invariance is related to the impossibility of efficient decentralised routeing when $\alpha \neq 2$ through the fact that shortcuts cannot make sufficient progress toward the destination when $\alpha>2$ (they are too short), while they cannot home in on small enough neighbourhoods of the destination when $\alpha<2$ (they are too long). Note that, while the number of connections per node is deterministic in Kleinberg's model, it is random (for both nearest neighbours and shortcuts) in our model and in Franceschetti and Meester's model.

A model very similar to ours was considered by Sharma and Mazumdar (2005), who used it to describe an ad hoc sensor network. The sensors are located at the points of a Poisson process and can communicate with nearby sensors through wireless links (corresponding to local contacts). In addition, it is possible to deploy a small number of wired links (corresponding to shortcuts); the question they addressed is that of how to place these wired links in order to enable efficient decentralised routeing.

In the analysis presented below, we ignore edge effects for ease of exposition. This is equivalent to considering distances as being defined on the torus obtained by identifying opposite edges of the square. 


\section{Efficiency of greedy routeing when $\alpha=2$}

When $\alpha=2$, we show that the following approximately greedy algorithm succeeds w.h.p. in reaching the destination in a number of hops which is polylogarithmic in $n$, the expected number of nodes.

Denote by $C(u, r)$ the circle of radius $r$ centred at node $u$. If there is no direct link from the source $s$ to the destination $t$, then the message is passed via intermediate nodes as follows. At each stage, the message carries the address (coordinates) of the destination $t$, as well as a radius $r$ which is initialised to $r(s, t)$, the distance between $s$ and $t$. Suppose that the message is currently at node $x$ and has radius $r>\sqrt{c \log n}$. (If $r \leq \sqrt{c \log n}$ then the node which updated $r$ would have contained $t$ in its local contact list and delivered the message immediately.) If node $x$ has a shortcut to some node $y \in A(t, r)$, where the annulus $A(t, r)$ is defined as $A(t, r)=C(t, r / 2) \backslash C(t, r / 4)$, then $x$ forwards the message to $y$. If there is more than one such node, then the choice can be arbitrary. Otherwise, it forwards the message to one of its local contacts which is closer to $t$ than itself. When a node $y$ receives a message, it updates $r$ to $r / 2$ if $r(y, t) \leq r / 2$, and leaves $r$ unchanged otherwise.

In other words, if $x$ can find a shortcut which reduces the distance to the destination by at least one-half but by no more than three-quarters, it uses such a shortcut. Otherwise, it uses a local contact to reduce the distance to the destination. In this sense, the algorithm is approximately greedy. The reason for considering such an algorithm rather than a greedy algorithm that would minimize the distance to the destination at each step is to preserve independence, which greatly simplifies the analysis. Note that if a greedy step from $x$ takes us to $y$ (i.e. of all nodes to which $x$ possesses a shortcut, $y$ is closest to $t$ ), then the conditional law of the point process in the circle $C(t, r(t, y))$ is no longer unit-rate Poisson. The fact that there are no shortcuts from $x$ to nodes within this circle biases the probability law and greatly complicates the analysis. Our approximate greedy algorithm gets around this problem.

Observe that if the message passes through a node $x$, the value of $r$ immediately after visiting $x$ lies between $r(x, t)$ and $2 r(x, t)$.

We have implicitly assumed that any node can find a local contact closer to $t$ than itself. We first show that this assumption holds w.h.p. if $c$ is chosen to be sufficiently large. Fix $c>0$ and $n \in \mathbb{N}$. For two points $x$ and $y$ in the square $[0, \sqrt{n}]^{2}$ and a realisation $\omega$ of the unit-rate Poisson process on the square, define the properties

$$
\mathcal{P}_{n}(x, y, \omega)=\{\exists u \in \omega: r(u, y)<r(x, y) \text { and } r(u, x) \leq \sqrt{c \log n}\}
$$

and

$$
\mathcal{P}_{n}(\omega)=\bigwedge_{\{(x, y): r(x, y) \geq \sqrt{c \log n}\}} \mathcal{P}_{n}(x, y, \omega) .
$$

Lemma 1. If $c>0$ is sufficiently large then $\mathrm{P}\left(\mathcal{P}_{n}(\cdot)\right) \rightarrow 1$ as $n \rightarrow \infty$.

Lemma 1 can be expressed as follows. With high probability, any two points $x$ and $y$ in the square $[0, \sqrt{n}]^{2}$, with $r(x, y)>\sqrt{c \log n}$, have the property that there is a point $u$ of the unitrate Poisson process within a distance $\sqrt{c \log n}$ of $x$ which is closer than $x$ to $y$. In particular, if $x$ and $y$ are themselves points of the Poisson process, then $u$ is a local contact of $x$ which is closer to $y$. The key point to note about Lemma 1 is that it gives a probability bound which is uniform over all such node pairs.

Proof of Lemma 1. Suppose that $r(x, t) \geq \sqrt{c \log n}$. Consider the circle $C_{1}$ of radius $\sqrt{c \log n}$ centred at $x$ and the circle $C_{2}$ of radius $r(x, t)$ centred at $t$. For any point $y \neq x$ 
in their intersection, $r(y, t)<r(x, t)$. Moreover, the intersection contains a sector of $C_{1}$ of angle $2 \pi / 3$. Denote this sector by $D_{1}$. Now consider a tessellation of the square $[0, \sqrt{n}]^{2}$ by small squares of side $\beta \sqrt{c \log n}$. Note that, for a sufficiently small geometrical constant $\beta$ that does not depend on $c$ or $n$ ( $\beta=\frac{1}{2}$ suffices), the sector $D_{1}$ fully contains at least one of the smaller squares. Hence, if every small square contains at least one point of the Poisson process, then every node at a distance greater than $\sqrt{c \log n}$ from $t$ can find at least one local contact which is closer to $t$. Number the small squares in some order and let $X_{i}$ denote the number of nodes in the $i$ th small square, for $i=1, \ldots, n /\left(\beta^{2} c \log n\right)$. The number of squares is assumed to be an integer for simplicity. Clearly, the $X_{i}$ are independent and identically distributed Poisson random variables with mean $\beta^{2} c \log n$. Hence, by the union bound, we obtain

$$
\mathrm{P}\left(\exists i: X_{i}=0\right) \leq \sum_{i=1}^{n /\left(\beta^{2} c \log n\right)} \mathrm{P}\left(X_{i}=0\right)=\frac{n}{\beta^{2} c \log n} \mathrm{e}^{-\beta^{2} c \log n},
$$

which goes to zero as $n$ tends to infinity, provided that $\beta^{2} c>1$. In particular, setting $c>4$ suffices since we can take $\beta=\frac{1}{2}$.

We now state the main result of this section.

Theorem 1. Consider the small-world random graph described above with $\alpha=2$, expected node degree $\bar{d}=1$, and $c>0$ sufficiently large, as required by Lemma 1. Then, the number of hops for message delivery between any pair of nodes is of order $\log ^{2} n$ w.h.p.

Remark 1. In Franceschetti and Meester (2003), a greedy algorithm was found to route a message in $O(\log n)$ hops whereas we require $O\left(\log ^{2} n\right)$ hops, as did Kleinberg (2000). The reason is that the average number of shortcuts per node is a constant in our model and in that of Kleinberg (2000), whereas in the model of Franceschetti and Meester (2003), when the links are restricted to have range between some fixed $\varepsilon$ and $\sqrt{n}$, the number of links per node is $O(\log n)$ (they do not distinguish between nearest neighbours and shortcuts).

Proof of Theorem 1. We first evaluate the normalisation constant $a_{n}$ by noting that the expected degree, $\bar{d}$, of a node located at the centre of the square satisfies

$$
\bar{d} \leq a_{n} \int_{\sqrt{c \log n}}^{\sqrt{n / 2}} x^{-2} 2 \pi x \mathrm{~d} x=\pi a_{n}(\log n-\log \log n-\log (2 c)),
$$

and so

$$
a_{n} \geq \frac{1}{\log n},
$$

for all $n$ sufficiently large, by the assumption that $\bar{d}=1$.

Next, we compute the probability of finding a suitable shortcut at each step of the greedy routeing algorithm. We think of the routeing algorithm as proceeding in phases. The value of $r$ is halved at the end of each phase. The value of $r$ immediately after the message reaches a node $x$ satisfies the relation $r(x, t) \in(r / 2, r]$ at each step of the routeing algorithm. We suppose that $r>k \sqrt{c \log n}$, for some large constant $k$.

Denote by $N_{A}$ the number of nodes in the annulus $A(t, r)$ and observe that $N_{A}$ is Poisson with mean $3 \pi r^{2} / 16$. The distance from $x$ to any of these nodes is bounded above by $3 r / 2$, and so the probability that a shortcut from $x$ is incident on a particular one of these nodes is 
bounded below by $a_{n}(3 r / 2)^{-2}$. Thus, conditional on $N_{A}$, the probability that $x$ has a shortcut to one of the $N_{A}$ nodes in $A(t, r)$ is bounded below by

$$
p\left(r, N_{A}\right)=1-\left(1-\frac{4 a_{n}}{9 r^{2}}\right)^{N_{A}} .
$$

If $x$ does not have such a shortcut, the message is passed via local contacts which are successively closer to $t$, and hence satisfy the same lower bound on the probability of a shortcut to $A(t, r)$. Consequently, the number of local steps, $L_{x}$, until a shortcut is found is bounded above by a geometric random variable with conditional mean $1 / p\left(r, N_{A}\right)$. Since $N_{A} \sim \operatorname{Po}\left(3 \pi r^{2} / 16\right)$, we obtain, by a standard application of the Chernoff bound,

$$
\mathrm{P}\left(N_{A} \leq \frac{\gamma r^{2}}{16}\right) \leq \exp \left(-\frac{(3 \pi-\gamma) r^{2}}{16}+\frac{\gamma r^{2}}{16} \log \frac{3 \pi}{\gamma}\right),
$$

for any $\gamma<3 \pi$.

Suppose first that $r \geq k \sqrt{c \log n}$, for some large constant $k$. Taking $\gamma=3 \pi / 2$, we obtain

$$
\mathrm{P}\left(N_{A} \leq \frac{3 \pi r^{2}}{32}\right) \leq \exp \left(-\frac{3 \pi k^{2} c \log n}{32}(1-\log 2)\right) .
$$

Now suppose that $N_{A}<3 \pi r^{2} / 32$. The number of local hops, $L_{x}$, to route the message from $x$ to $A$ is bounded above by the number of nodes outside $A$, since the distance to $t$ is strictly decreasing after each hop. Hence,

$$
\mathrm{E}\left[L_{x} \mid N_{A}<\frac{3 \pi r^{2}}{32}\right] \leq n-\operatorname{area}(A) \leq n .
$$

Next, if $N_{A} \geq 3 \pi r^{2} / 32$, then we have, by (2) and (1),

$$
p\left(r, N_{A}\right) \geq 1-\exp \left(-\frac{\pi a_{n}}{24}\right) \geq 1-\exp \left(-\frac{\pi}{24 \log n}\right) \geq \frac{\pi}{48 \log n},
$$

where the last inequality holds for all $n$ sufficiently large. Since the number of hops to reach $A$ is bounded above by a geometric random variable with mean $1 / p\left(r, N_{A}\right)$, we have

$$
\mathrm{E}\left[L_{x} \mid N_{A} \geq \frac{3 \pi r^{2}}{32}\right] \leq \frac{48}{\pi} \log n .
$$

Finally, we obtain, from (3), (4), and (5),

$$
\mathrm{E}\left[L_{x}\right] \leq n \exp \left(-\frac{3 \pi k^{2} c(1-\log 2)}{32} \log n\right)+\frac{48}{\pi} \log n .
$$

The first term in the sum in (6) can be made arbitrarily small by choosing $k$ large enough, so $\mathrm{E}\left[L_{x}\right]=O(\log n)$. It can also be seen from the arguments above that $L_{x}=O(\log n)$ w.h.p. In other words, while $r \geq k \sqrt{c \log n}$, the number of hops during each phase is of order $\log n$. Moreover, the number of such phases is of order $\log n$ since the initial value of $r$ is at most $\sqrt{2 n}$, and $r$ halves at the end of each phase.

Hence, the total number of hops until $r<k \sqrt{c \log n}$ is of order $\log ^{2} n$. Once the message reaches a node $x$ with $r(x, t)<k \sqrt{c \log n}$, the number of additional hops to reach $t$ is bounded above by the total number of nodes in the circle $C(t, k \sqrt{c \log n})$. By using the Chernoff bound for a Poisson random variable, it can be shown that this number is of order $\log n$ w.h.p. This completes the proof of Theorem 1 . 


\section{The impossibility of efficient routeing when $\alpha \neq 2$}

We now show that if $\alpha<2$ then no decentralised algorithm can route between arbitrary source-destination pairs in a time which is polylogarithmic in $n$. In fact, the number of routeing hops is polynomial in $n$ with some fractional power that depends on $\alpha$.

We now make precise what we mean by a decentralised routeing algorithm. As specified earlier, each node knows the locations of all its local contacts at distance $\sqrt{c \log n}$ and all its shortcut neighbours, as well as other nodes (if any) from which shortcuts are incident to it. A routeing algorithm specifies a (possibly random) sequence of nodes

$$
s=x_{0}, x_{1}, \ldots, x_{k}=t, x_{k+1}=t, \ldots,
$$

where the only requirement is that each node $x_{i}$ is chosen from among the local or shortcut contacts of nodes $\left\{x_{0}, \ldots, x_{i-1}\right\}$. (This is the same definition as used by Kleinberg (2000).)

Theorem 2. Consider the small-world random graph described above with $\alpha<2$ and arbitrarily large constants $c$ and $\bar{d}$. Suppose that the source $s$ and destination t are chosen uniformly at random from the node set. Then the number of hops for message delivery in any decentralised algorithm exceeds $n^{\gamma}$ w.h.p., for any $\gamma<(2-\alpha) / 6$.

It is not important that the source and destination are chosen uniformly but only that the distance between them is of order $n^{a}$ w.h.p., for some $a>0$.

Proof of Theorem 2. We first evaluate the normalisation constant $a_{n}$ by noting that the expected degree satisfies

$$
\bar{d} \geq a_{n} \int_{\sqrt{c \log n}}^{\sqrt{n} / 2} x^{-\alpha} 2 \pi x \mathrm{~d} x=\frac{2 \pi a_{n}}{2-\alpha}\left(\frac{n^{(2-\alpha) / 2}}{2^{2-\alpha}}-(c \log n)^{(2-\alpha) / 2}\right),
$$

which, on simplification, yields

$$
a_{n} \leq \frac{4 \bar{d}}{n^{(2-\alpha) / 2}},
$$

for all $n$ sufficiently large. Note that $a_{n}$ is an upper bound on the probability that there is a shortcut between any pair of nodes.

Suppose that the source $s$ and destination $t$ are chosen uniformly from all nodes in $[0, \sqrt{n}]^{2}$. Fix $\delta \in\left(\gamma, \frac{1}{2}\right)$ and define $C_{\delta}=C\left(t, n^{\delta}\right)$ to be the circle of radius $n^{\delta}$ centred at $t$. It is clear that, for any $\varepsilon>0$, the distance $r\left(s, C_{\delta}\right)$ from $s$ to the circle $C_{\delta}$ is bigger than $n^{1 / 2-\varepsilon}$ w.h.p. Suppose now that this inequality holds, but that there is a routeing algorithm which can route from $s$ to $t$ in fewer than $n^{\gamma}$ hops. Denote by $s=x_{0}, x_{1}, \ldots, x_{m}=t$ the sequence of nodes visited by the routeing algorithm, with $m \leq n^{\gamma}$. We claim that there must be a shortcut from at least one of the nodes $x_{0}, x_{1}, \ldots, x_{m-1}$ to the set $C_{\delta}$. Indeed, if there is no such shortcut, then $t$ must be reached starting from some node outside $C_{\delta}$ and using only local links. Since the length of each local link is at most $\sqrt{c \log n}$ and the number of hops is at most $n^{\gamma}$, the total distance traversed by local hops is strictly smaller than $n^{\delta}$ (for large enough $n$, by the assumption that $\delta>\gamma$ ), which yields a contradiction. We now estimate the probability that there is a shortcut from one of the nodes $x_{0}, \ldots, x_{m-1}$ to the set $C_{\delta}$.

The number of nodes in the circle $C_{\delta}$, denoted by $N_{C}$, is Poisson with mean $\pi n^{2 \delta}$, so $N_{C}<4 n^{2 \delta}$ w.h.p. Now, by (7) and the union bound we obtain

$$
\mathrm{P}\left(\exists \text { shortcut between } u \text { and } C_{\delta} \mid N_{C}<4 n^{2 \delta}\right) \leq 16 \bar{d} n^{(4 \delta+\alpha-2) / 2},
$$


for any node $u$. Applying this bound repeatedly for each of the nodes $x_{0}, x_{1}, \ldots, x_{m-1}$ generated by the routeing algorithm, we obtain

$$
\mathrm{P}\left(\exists \text { shortcut to } C_{\delta} \text { within } n^{\gamma} \text { hops } \mid N_{C}<4 n^{2 \delta}\right) \leq 16 \bar{d} n^{(2 \gamma+4 \delta+\alpha-2) / 2} .
$$

Now $\gamma<(2-\alpha) / 6$ by assumption, and $\delta>\gamma$ can be chosen arbitrarily. In particular, we can choose $\delta$ so that $2 \gamma+4 \delta+\alpha-2$ is strictly negative, in which case the conditional probability of a shortcut to $C_{\delta}$ goes to zero as $n \rightarrow \infty$. Since $\mathrm{P}\left(N_{C} \geq 4 n^{2 \delta}\right)$ also goes to zero, we conclude that the probability of finding a route from $s$ to $t$ with fewer than $n^{\gamma}$ hops also goes to zero. This concludes the proof of Theorem 2.

Remark 2. Theorem 2 continues to hold if we assume one-step lookahead. By this we mean that when a node decides where to send the message at the next step, it can not only use the locations of all its local and shortcut contacts, but also the locations of their contacts. All this means is that after visiting $n^{\gamma}$ nodes, the algorithm has knowledge of $O\left(n^{\gamma} \log n\right)$ nodes. If none of these nodes has a shortcut into the set $C_{\delta}$, which is the case w.h.p., then the arguments above still apply. The same is true for $k$-step lookahead, for any constant $k$.

Theorem 3. Consider the small-world random graph described above with $\alpha>2$ and arbitrarily large constants $c$ and $\bar{d}$. Suppose that the source $s$ and destination t are chosen uniformly at random from the node set. Then the number of hops for message delivery in any decentralised algorithm exceeds $n^{\gamma}$ w.h.p., for any $\gamma<(\alpha-2) / 2(\alpha-1)$.

Proof. For a node $u$, the probability that a randomly generated shortcut has length greater than $r$ is bounded above by

$$
\left(\int_{r}^{\infty} x^{-\alpha} 2 \pi x \mathrm{~d} x\right)\left(\int_{\sqrt{c \log n}}^{\sqrt{n} / 2} x^{-\alpha} 2 \pi x \mathrm{~d} x\right)^{-1} \leq \text { constant } \times r^{2-\alpha}(\log n)^{(\alpha-2) / 2},
$$

for all $n$ sufficiently large. Since there are $2 \bar{d}$ shortcuts per node on average, the probability that two nodes $u$ and $v$ separated by a distance $r$ or more possess a shortcut between them is bounded above by the same function, but with the constant suitably modified.

Now, for randomly chosen nodes $s$ and $t, r(s, t)>n^{1 / 2-\varepsilon}$ w.h.p., for any $\varepsilon>0$. Hence, there can be a path of length $n^{\gamma}$ hops between $s$ and $t$ only if at least one of the hops is a shortcut of length $n^{1 / 2-\varepsilon-\gamma}$ or more. By the above and the union bound, the probability of there being such a shortcut is bounded above by

$$
\text { constant } \times n^{\gamma}\left(n^{1 / 2-\varepsilon-\gamma}\right)^{2-\alpha}(\log n)^{(\alpha-2) / 2} .
$$

The exponent of $n$ in the above expression is

$$
\frac{2-\alpha}{2}(1-2 \varepsilon)+\gamma(\alpha-1)
$$

The exponent above is negative for sufficiently small $\varepsilon>0$ provided that

$$
\gamma<\frac{\alpha-2}{2(\alpha-1)}
$$

In other words, if this inequality is satisfied, then the probability of finding a route with fewer than $n^{\gamma}$ hops goes to zero as $n \rightarrow \infty$. This establishes the claim of the theorem. 


\section{References}

Barbour, A. D. And Reinert, G. (2001). Small worlds. Random Structures Algorithms 19, 54-74.

Benjamini, I. AND Berger, N. (2001). The diameter of long-range percolation clusters on finite cycles. Random Structures Algorithms 19, 102-111.

Benjamini, I., Kesten, H., Peres, Y. and Schramm, O. (2004). Geometry of the uniform spanning forest: transitions in dimensions 4, 8, 12, ... Ann. Math. 160, 465-491.

Coppersmith, D., GAMARnik, D. AND SviridenKo, M. (2002). The diameter of a long-range percolation graph. Random Structures Algorithms 21, 1-13.

ERdős, P. And RÉnyI, A. (1959). On random graphs. I. Publ. Math. Debrecen 6, 290-297.

Franceschetti, M. and Meester, R. (2003). Navigation in small world networks, a scale-free continuum model. Tech. Rep. UCB/ERL M03/33, EECS Department, University of California, Berkeley.

Kleinberg, J. (2000). The small-world phenomenon: an algorithmic perspective. In Proc. 32nd Annual ACM Symp. Theory Comput., ACM, New York, pp. 163-170.

Milgram, S. (1967). The small world problem. Psychology Today 2, 60-67.

Penrose, M. (2003). Random Geometric Graphs. Oxford University Press.

Sharma, G. and Mazumdar, R. R. (2005). Hybrid sensor networks: a small world. In Proc. 6th ACM Internat. Symp. Mobile Ad Hoc Networking Comput., ACM, New York, pp. 366-377.

Watts, D. J. And Strogatz, S. H. (1967). Collective dynamics of small world networks. Nature 393, 440-442. 